\title{
A generalized network model for polymeric liquids
}

\author{
R. J.J. Jongschaap, H. Kamphuis, and D. K. Doeksen \\ Rheology Group, Department of Applied Physics, Twente University of Technology, Enschede (The Netherlands)
}

\begin{abstract}
A kinetic model was developed for relating the molecular structure and the rheological behaviour of polymer-like materials in which bonds are being created and broken. In particular, the stress contribution of molecules that are not a part of the network was taken account of. In two limiting cases the model introduced here is identical to the transient-network model and the bead-spring model, respectively. Model predictions were interpreted and compared with measurements on an L.D.-polyethylene melt, available from literature, and an S.I.S.-blockcopolymer solution. In both cases information was obtained about the network and the kinetic processes occurring in a state of rest and during steadyshear flow, assuming the model to be valid.
\end{abstract}

Key words: Constitutive equation, transient-network model, bead-spring model, molecular structure, polymer

List of symbols

a kinetic parameter

$c$ molecular density

$D$ rate-of-strain tensor

$f_{i} \quad$ fraction of segments of type $i$

$G_{i} \quad$ storage moduli

$g, g_{i}$ creation functions

$h, h_{i}$ annihilation functions

$H$ spring constant

$k \quad$ Boltzmann's constant

$L \quad$ velocity gradient tensor

$N$ total segment density

$N_{i} \quad$ density of segments of type $i$

$q$ segment vector

$t$ time

$T$ absolute temperature

$\boldsymbol{T}$ macroscopic stress tensor

$x \quad$ structural factor

\section{Greek symbols}

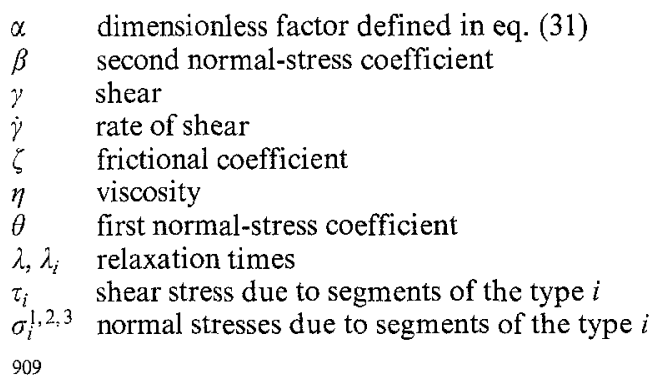

$\psi \quad$ segment distribution function normalized to unity

$\Psi \quad$ segment distribution function normalized to $N$

\section{Introduction}

The transient-network model, originally proposed by Green and Tobolsky [1], Lodge [2], and Yamamoto [3], has proved to be a good starting point for the development of theories about the rheological behaviour of polymer melts and solutions. For a discussion of some of these theories and of the basic assumptions of the transient-network model we refer to [4], Ch. 15. Here we just mention that in the model the polymer molecules are assumed to form a network in which segments are lost and created during flow. The stress is assumed to be the sum of the contributions from all segments of 
the network at the present time. All macromolecular chains not belonging to the network are assumed to give no contribution to the stress. Furthermore, it is assumed that the distribution function for the network segments at the moment of creation is identical to the . equilibrium distribution function of a free chain.

In the present paper a generalized transient-network theory is proposed in which these assumptions are modified. A general description of this theory and an analysis of its predictions for the linear viscoelastic behaviour and the behaviour under steady and transient shear flow conditions are given in section 2 . In section 3 these predictions are compared with experimental results.

\section{Theory}

\subsection{General description}

The system is assumed to consist of two types of segments: 'fixed segments', which, as usual, form a network, and 'free segments', which behave like chains in bead-spring models $[4,5,6]$ of polymer solutions (fig. 1). Transitions between the two types of segments may take place. As opposed to the original transient-network theories, the distribution function at the moment of formation of fixed segments is not assumed

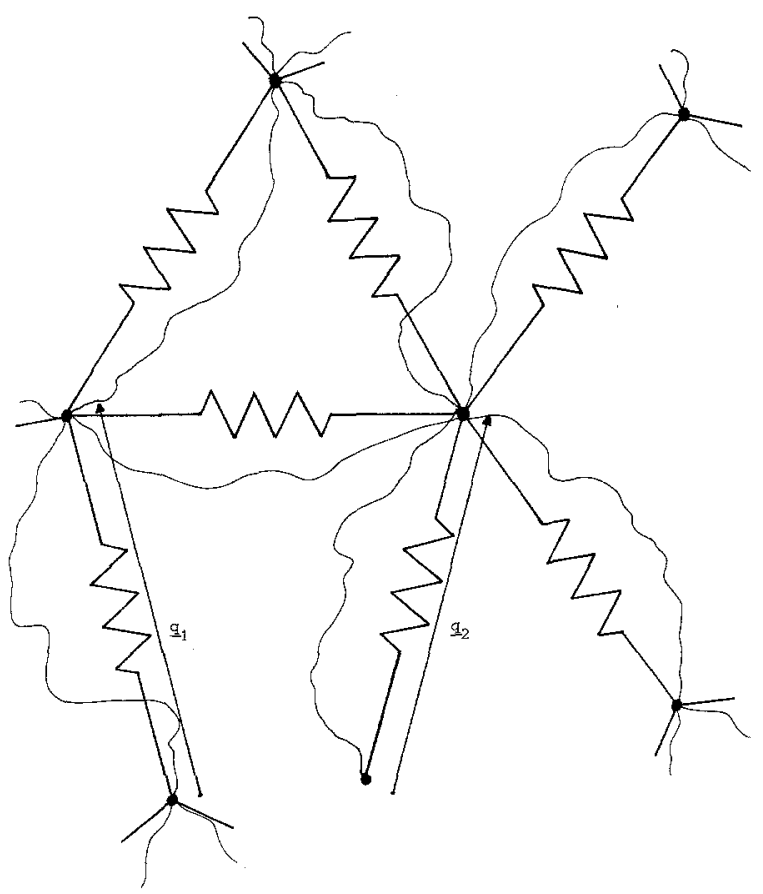

Fig. 1. Polymer network containing fixed and free segments, identified by index 1 and 2, respectively. to be the equilibrium distribution, but, instead, it is proportional to the distribution of the free segments. Furthermore, the total stress tensor in the system is not only determined by the fixed but also by the free segments.

For the modelling of the free and the fixed segments, in principle several levels of refinement are possible. The segments may have various 'complexities' [7] or be able to perform several modes of motions [5]. In this paper, in order to concentrate on the essential features of the model, we will only discuss the simplest case, in which just one type of fixed segments exists and the free segments may be modelled as elastic dumbbells (cf. ref. [4], Ch. 10).

\subsection{The constitutive equation}

We consider an incompressible fluid with $c$ macromolecules per unit volume. As already stated in section 2.1 , it will be assumed that this system contains identical segments, that may be either fixed or free. Let $N_{1}$ be the concentration of fixed and $N_{2}$ the concentration of free segments. We have

$$
N_{1}+N_{2}=N \text {, }
$$

in which $N$, the total segment concentration, is a constant.

Each segment may be modelled as a Gaussian spring with an equilibrium distribution *)

$$
\psi_{0}=\left(\frac{H}{2 \pi k T}\right)^{3 / 2} \exp \left(-\frac{H \boldsymbol{q}^{2}}{2 k T}\right)
$$

where $\boldsymbol{q}$ is the end-to-end vector and $H$ a spring constant.

The actual distribution functions of the fixed and free segments will be denoted by $\psi_{1}(\boldsymbol{q}, t)$ and $\psi_{2}(\boldsymbol{q}, t)$, respectively. The distribution functions $\psi_{0}, \psi_{1}$, and $\psi_{2}$ are normalized to unity:

$$
\int \psi_{i}(\boldsymbol{q}, t) d^{3} \boldsymbol{q}=1, \quad(i=0,1,2) .
$$

The total numbers, per unit volume, of fixed $(i=1)$ and free segments $(i=2)$ that are found within the configuration range $d^{3} \boldsymbol{q}$ around $\boldsymbol{q}$ are represented by

$$
\Psi_{i}(\boldsymbol{q}, t) \equiv N_{i} \psi_{i}(\boldsymbol{q}, t), \quad(i=1,2) .
$$

The balance equations that govern the time dependence of the distribution functions will be of the usual form of equations of continuity in configuration space, supplemented by creation and annihilation terms

*) Here and elsewhere in this paper a subscript 0 is used to denote the equilibrium form or value of a quantity. 
describing the transition of one type of segments to the other. For the fixed segments we have:

$$
\frac{\partial \Psi_{1}}{\partial t}=-\operatorname{div}\left(\Psi_{1} \dot{\boldsymbol{q}}_{1}\right)+g \psi_{2}-h \psi_{1},
$$

and for the free segments:

$$
\frac{\partial \Psi_{2}}{\partial t}=-\operatorname{div}\left(\Psi_{2} \dot{\boldsymbol{q}}_{2}\right)-g \psi_{2}+h \psi_{1} .
$$

In these equations the creation and annihilation functions $g$ and $h$ may depend on averaged properties of the system, such as segment concentrations, stresses, and the rate of strain. They are, however, independent of the configuration vector $\boldsymbol{q}$.

From (5) we see that, as stated before, the distribution function of the fixed segments, at the instant of creation, is proportional to the distribution $\psi_{2}$ of the free segments and not, like in most transient-network theories, to the equilibrium distribution (2).

With regard to the rates of change, $\dot{\boldsymbol{q}}_{1}$ and $\dot{\boldsymbol{q}}_{2}$, of the end-to-end vectors of the fixed and the free segments, we make the assumption that the fixed segments deform affinely with the macroscopic flow, described by $\boldsymbol{L}$, the macroscopic velocity gradient tensor, and that the free segments move like elastic dumbbells on which viscous and Brownian forces are acting. So

$$
\dot{q}_{1}=L \cdot q_{1}
$$

and

$$
\dot{\boldsymbol{q}}_{2}=\boldsymbol{L} \cdot \boldsymbol{q}_{2}-\frac{2 k T}{\zeta} \frac{\partial}{\partial \boldsymbol{q}} \ln \frac{\psi_{2}}{\psi_{0}}
$$

where $k$ is Boltzmann's constant, $T$ the absolute temperature, $\zeta$ a friction coefficient, and $\psi_{0}$ the equilibrium distribution, given by eq. (2).

The contribution of the polymer molecules to the stress tensor is assumed to be given by

$$
T_{p}=T_{1}+T_{2}
$$

where the partial stresses $\boldsymbol{T}_{1}$ and $\boldsymbol{T}_{2}$, due to the fixed and the free segments are given by, respectively,

$$
\boldsymbol{T}_{i}=-N_{i} k T \mathbf{1}+N_{i} H\langle\boldsymbol{q} \boldsymbol{q}\rangle_{i}, \quad(i=1,2) .
$$

This is the usual expression for Gaussian segments. In this expression $H$ is the parameter defined in eq. (2) and the brackets denote an averaging with respect to the distribution function $\psi_{i}$ :

$$
\langle A\rangle_{i} \equiv \int A(\boldsymbol{q}) \psi_{i}(\boldsymbol{q}, t) d^{3} \boldsymbol{q} .
$$

Similarly, averages with respect to the equilibrium distribution $\psi_{0}$ are denoted by $\langle\cdots\rangle_{0}$. The pressure term in (10) was chosen in such a way that, since $\langle\boldsymbol{q} \boldsymbol{q}\rangle_{0}=\frac{k T}{H} \mathbf{1}$, at equilibrium we have

$$
T_{10}=T_{20}=T_{0}=\mathbf{0} .
$$

From the microscopic equations $(5-8)$ we derive equations for the time-dependence of the segment concentrations $N_{1}$ and $N_{2}$ and the partial stresses $\boldsymbol{T}_{1}$ and $\boldsymbol{T}_{2}$. First, by integrating (5) and (6) over all configurations we obtain the kinetic expressions

$$
\frac{d N_{1}}{d t}=-\frac{d N_{2}}{d t}=g-h
$$

Secondly, by multiplying (5) and (6) by $\boldsymbol{q} q$, substituting (7) and (8), and integrating over all configurations, we obtain:

$$
\frac{\mathfrak{D}}{\mathfrak{D} t} N_{1}\langle\boldsymbol{q} \boldsymbol{q}\rangle_{1}=g\langle\boldsymbol{q} \boldsymbol{q}\rangle_{2}-h\langle\boldsymbol{q} \boldsymbol{q}\rangle_{1}
$$

and

$$
\begin{aligned}
\frac{\mathfrak{D}}{\mathfrak{D} t} N_{2}\langle\boldsymbol{q} \boldsymbol{q}\rangle_{2}= & \frac{4 k T}{\zeta} N_{2}\left(\mathbf{1}-\frac{H}{k T}\langle\boldsymbol{q} \boldsymbol{q}\rangle_{2}\right) \\
& -g\langle\boldsymbol{q} \boldsymbol{q}\rangle_{2}+h\langle\boldsymbol{q} \boldsymbol{q}\rangle_{1},
\end{aligned}
$$

where $\mathfrak{D} / \mathfrak{D} t$ denotes the so-called upper convected or Oldroyd derivative defined as

$$
\frac{\mathfrak{D}}{\mathfrak{D} t} \boldsymbol{A} \equiv \frac{d}{d t} \boldsymbol{A}-\boldsymbol{L} \cdot \boldsymbol{A}-\boldsymbol{A} \cdot \boldsymbol{L}^{T}
$$

Using (10) and (13), we obtain from (14) and (15) the following constitutive equations for the partial stresses $\boldsymbol{T}_{1}$ and $\boldsymbol{T}_{2}$ :

$$
\begin{aligned}
& \frac{\mathfrak{D}}{\mathfrak{D} t} \boldsymbol{T}_{1}+h_{1} \boldsymbol{T}_{1}-g_{2} \boldsymbol{T}_{2}=2 N_{1} k T \boldsymbol{D} \\
& \frac{\mathfrak{D}}{\mathfrak{D} t} \boldsymbol{T}_{2}+\left(g_{2}+\lambda^{-1}\right) \boldsymbol{T}_{2}-h_{1} \boldsymbol{T}_{1}=2 N_{2} k T \boldsymbol{D} .
\end{aligned}
$$

In these equations we used the notations

$$
g_{i} \equiv g / N_{i}, \quad h_{i} \equiv h / N_{i}, \quad(i=1,2),
$$

which are material functions with the dimension of a reciprocal time,

$$
\lambda \equiv \frac{\zeta}{4 H},
$$

the usual time constant of the elastic-dumbbell model, and

$$
D \equiv \frac{1}{2}\left(L+L^{T}\right),
$$

the rate-of-strain tensor.

The expressions (9), (13), (17), and (18) form a set of macroscopic equations which describe the rheological 
behaviour of the system. In order to obtain specific results, further assumptions about the functions $g$ and $h$ have to be made. First, however, some general properties of the model will be discussed.

\subsection{Limiting cases}

In the case that the friction coefficient $\zeta$ approaches zero, the segments will adopt their equilibrium distribution immediately after formation. By (2) and (10) we then have $\boldsymbol{T}_{2}=\mathbf{0}$, so $\boldsymbol{T}_{p}=\boldsymbol{T}_{1}$, since from (8) it follows that $\psi_{2}=\psi_{0}$. These are just the assumptions of the usual transient-network theories. Indeed, in that case eq. (17) reduces to

$$
\frac{\mathfrak{D}}{\mathfrak{D} t} \boldsymbol{T}_{p}+h_{1} \boldsymbol{T}_{p}=2 N_{1} k T \boldsymbol{D},
$$

which is the constitutive equation of transient-network theory in case of one type (complexity) of segments (see, for instance, [8], eqs. (23) and (32)). Eq. (13) being used, (22) may also be written in the form

$$
g_{1}^{-1} \frac{\mathfrak{D}}{\mathfrak{D} t}\left(\frac{\boldsymbol{T}_{p}}{N_{1} k T}\right)+\left(\frac{\boldsymbol{T}_{p}}{N_{1} k T}\right)=2 g_{1}^{-1} \boldsymbol{D} .
$$

In the present paper the functions $g$ and $h$ will be chosen such that eqs. (13) and (23) are equivalent to a model first proposed by Marrucci $[9,10]$. From the results of [8] it can be shown that in this case the appropriate forms of these functions are:

$$
\begin{aligned}
& g=g_{0} x^{-0.4}, \\
& h=g\left(a \sqrt{\frac{\operatorname{tr} T_{1}}{2 N_{1} k T}}-\frac{1}{x}+2\right),
\end{aligned}
$$

where $g_{0}$ is the equilibrium value of the function $g, x$ is a structural variable defined as $x \equiv N_{1} / N_{10}$, and a is a constant.

In the second limiting case of our model, $N_{1}=0$ and $g=h=0$, we have $N_{2}=N, \boldsymbol{T}_{1}=\mathbf{0}$, and $\boldsymbol{T}_{p}=\boldsymbol{T}_{2}$. In that case (18) reduces to

$$
\lambda \frac{\mathfrak{D}}{\mathfrak{D} t} \boldsymbol{T}_{p}+\boldsymbol{T}_{p}=2 N k T \lambda \boldsymbol{D},
$$

which corresponds with the elastic dumbbell model (see [4], eq. (10.4-4)). The choice of the material parameters that corresponds to the two limiting cases will be discussed in the following section.

\subsection{Linear viscoelasticity}

The model predictions of the linear viscoelastic behaviour is determined by the linearized form of eqs. (17) and (18). From (13) and (19) it follows that

$$
g_{20} N_{20}=h_{10} N_{10} \text {. }
$$

Using (1), (9), and (27), from (17) and (18) one obtains the complex viscosity $\eta^{*}(i \omega)$ :

$$
\begin{aligned}
& \eta^{*}(i \omega)=\frac{\lambda_{1} G_{1}}{1+i \omega \lambda_{1}}+\frac{\lambda_{2} G_{2}}{1+i \omega \lambda_{2}}, \\
& \lambda_{1,2}=\frac{1}{2 h_{10}}\left(\alpha \pm \sqrt{\alpha^{2}-4 \lambda h_{10}}\right), \\
& G_{1,2}=\frac{N k T}{2}\left(1 \pm \frac{\alpha+2\left(f_{10}-1\right)}{\sqrt{\alpha^{2}-4 \lambda h_{10}}}\right)
\end{aligned}
$$$$
\text { with }
$$

In these expressions

$$
\alpha \equiv 1+\frac{\lambda h_{10}}{1-f_{10}}
$$

and

$$
f_{10} \equiv \frac{N_{10}}{N},
$$

the equilibrium value of $f_{1}=N_{1} / N$, the fraction of fixed segments. Note that by (32) the structural factor $x=f_{1} / f_{10}$.

We see that the linear viscoelastic response of our model is determined by two relaxation times, $\lambda_{1}$ and $\lambda_{2}$, and two moduli, $G_{1}$ and $G_{2}$. These constants are related to the model parameters $\lambda, h_{10}, f_{10}$, and $N$ by the expressions $(29-32)$.

In the limiting cases, discussed in section 2.2, we obtain from (29-32), for $\lambda=0$ (transient-network model):

$$
\begin{array}{ll}
\lambda_{1}=h_{10}^{-1}, & G_{1}=N_{10} k T, \\
\lambda_{2}=0, & G_{2}=N_{20} k T,
\end{array}
$$

and for $f_{10}=0$ (elastic-dumbbell model):

$$
\begin{array}{ll}
\lambda_{1}=h_{10}^{-1}, & G_{1}=0, \\
\lambda_{2}=\lambda, & G_{2}=N k T .
\end{array}
$$

After substitution in (28) these results give expressions for the complex viscosity that are in accordance with the constitutive equations (22) and (26).

\subsection{Shear flow}

In shear flow with a velocity field $v_{x}=\dot{\gamma}(t) y, v_{y}=0$, $v_{z}=0$, the partial stresses $\boldsymbol{T}_{i}(i=1,2)$ will in general be of the form

$$
\left(\boldsymbol{T}_{i}\right)=\left(\begin{array}{lll}
\sigma_{i}^{1} & \tau_{i} & 0 \\
\tau_{i} & \sigma_{i}^{2} & 0 \\
0 & 0 & \sigma_{i}^{3}
\end{array}\right), \quad(i=1,2) .
$$


From (17) and (18) a set of differential equations can be obtained by which, together with the kinetic equation (13), for a given deformation history the components of the partial stresses (37) and the segment concentrations $N_{1}(t)$ and $N_{2}(t)$ are determined:

$$
\begin{aligned}
& \dot{\sigma}_{1}^{1}=g_{2} \sigma_{2}^{1}-h_{1} \sigma_{1}^{1}+2 \dot{\gamma} \tau_{1}, \\
& \dot{\sigma}_{1}^{2}=g_{2} \sigma_{2}^{2}-h_{1} \sigma_{1}^{2}, \\
& \dot{\sigma}_{1}^{3}=g_{2} \sigma_{2}^{3}-h_{1} \sigma_{1}^{3}, \\
& \dot{\tau}_{1}=g_{2} \tau_{2}-h_{1} \tau_{1}+\dot{\gamma}\left(\sigma_{1}^{2}+N_{1} k T\right), \\
& \dot{\sigma}_{2}^{1}=h_{1} \sigma_{1}^{1}-\left(g_{2}+\frac{1}{\lambda}\right) \sigma_{2}^{1}+2 \dot{\gamma} \tau_{2}, \\
& \dot{\sigma}_{2}^{2}=h_{1} \sigma_{1}^{2}-\left(g_{2}+\frac{1}{\lambda}\right) \sigma_{2}^{2}, \\
& \dot{\sigma}_{2}^{3}=h_{1} \sigma_{1}^{3}-\left(g_{2}+\frac{1}{\lambda}\right) \sigma_{2}^{3}, \\
& \dot{\tau}_{2}=h_{1} \tau_{1}-\left(g_{2}+\frac{1}{\lambda}\right) \tau_{2}+\dot{\gamma}\left(\sigma_{2}^{2}+N_{2} k T\right), \\
& \dot{N}_{1}=g_{2} N_{2}-h_{1} N_{1} .
\end{aligned}
$$

We define the dimensionless variables (cf. [9]),

$$
\begin{aligned}
\bar{\sigma}_{i}^{1} & \equiv \frac{a^{2} \sigma_{i}^{1}}{2 N_{10} k T} \quad(i=1,2), \\
\bar{\tau}_{i} & \equiv \frac{a \tau_{i}}{N_{10} k T} \quad(i=1,2), \\
\bar{\sigma}^{1} & \equiv \bar{\sigma}_{1}^{1}+\bar{\sigma}_{2}^{1}, \\
\bar{\tau} & \equiv \bar{\tau}_{1}+\bar{\tau}_{2}, \\
\bar{\gamma} & \equiv \frac{a \dot{\gamma}}{h_{10}}, \\
\bar{t} & \equiv t h_{10} .
\end{aligned}
$$

From eqs. (24-25), (38-46), together with (1), noting that as a consequence of initial condition (12) $\sigma_{1}^{2}=\sigma_{2}^{2}$ $=\sigma_{1}^{3}=\sigma_{2}^{3}=0$, the following set of differential equations for these variables can be obtained:

$$
\begin{aligned}
& \frac{d x}{d \bar{t}}=x^{-0.4}\left(\frac{1}{x}-1-\sqrt{(\bar{\sigma} 1 / x)}\right), \\
& \frac{d \bar{\sigma}_{1}^{1}}{d \bar{t}}=\frac{\left(f_{10} \bar{\sigma}^{1}-\bar{\sigma}_{1}^{1} / x\right)}{\left(1-f_{10} x\right) x^{0.4}}+\bar{\gamma} \bar{\tau}_{1}+\frac{\bar{\sigma}_{1}^{1}}{x} \frac{d x}{d \bar{t}}, \\
& \frac{d \bar{\tau}_{1}}{d \dot{t}}=\frac{\left(f_{10} \bar{\tau}-\bar{\tau}_{1} / x\right)}{\left(1-f_{10} x\right) x^{0.4}}+\bar{\gamma} x+\frac{\bar{\tau}_{1}}{x} \frac{d x}{d \bar{t}}, \\
& \frac{d \bar{\sigma}^{1}}{d t}=\frac{\left(\bar{\sigma}_{1}^{1}-\bar{\sigma}^{1}\right)}{\lambda h_{10}}+\bar{\gamma} \bar{\tau}, \\
& \frac{d \bar{\tau}}{d \bar{t}}=\frac{\left(\bar{\tau}_{1}-\bar{\tau}\right)}{\lambda h_{10}}+\frac{\bar{\gamma}}{f_{10}} .
\end{aligned}
$$

The initial conditions follow from (12) and (32). Note that the solutions of these equations only depend on two dimensionless parameters: the equilibrium fraction of fixed segments and the ratio of characteristic times, $\lambda h_{10}$. In a stationary shear flow all time derivatives equal zero, and one readily obtains the dimensionless viscometric functions

$$
\begin{aligned}
& \bar{\eta}=\eta \frac{h_{10}}{N_{10} k T}, \\
& \bar{\theta} \equiv \theta \frac{h_{10}^{2}}{2 N_{10} k T}
\end{aligned}
$$

and

$$
\bar{\beta} \equiv \beta \frac{h_{10}^{2}}{2 N_{10} k T},
$$

where $\eta(\dot{\gamma}) \equiv T_{x y} / \dot{\gamma}, \quad \theta(\dot{\gamma}) \equiv\left(T_{x x}-T_{y y}\right) / \dot{\gamma}^{2}$, and $\beta(\dot{\gamma})$ $\equiv\left(T_{y y}-T_{z z}\right) / \dot{\gamma}^{2}$, in terms of the fraction $f_{1}=N_{1} / N$ of fixed segments:

$$
\begin{aligned}
& \bar{\eta}=\frac{h_{10}}{f_{10}}\left(\frac{f_{1}}{h_{1}}+\frac{\lambda}{1-f_{1}}\right), \\
& \bar{\theta}=\frac{h_{10}^{2}}{f_{10}}\left(\frac{f_{1}}{h_{1}^{2}}+\frac{\lambda f_{1}}{h_{1}\left(1-f_{1}\right)}+\frac{\lambda f_{10}}{h_{10}\left(1-f_{1}\right)} \bar{\eta}\right), \\
& \bar{\beta}=0 .
\end{aligned}
$$

In the limiting cases $\lambda=0$ and $f_{1}=0$ these expressions reduce to the corresponding results of the transientnetwork model and the bead-spring model, respectively.

The values of $f_{1}$ and $h_{1}$ in (61) and (62) are determined by the equation $g=h$, which follows from (13), and the specific form (25) of the function $h$. In this way we obtain the following equations:

$$
\begin{aligned}
& a \dot{\gamma} \sqrt{\frac{\lambda^{2}}{\left(1-f_{1}\right)^{2}}+\frac{2 \lambda}{h_{1}\left(1-f_{1}\right)}+\frac{1}{h_{l}^{2}}}-\frac{f_{10}}{f_{1}}+1=0, \\
& h_{1}=h_{10}\left(\frac{f_{1}}{f_{10}}\right)^{-1.4} .
\end{aligned}
$$

So, for given values of the material parameters $a, \lambda, h_{10}$, and $f_{10}$ the functions $x(\dot{\gamma}), f_{1}(\dot{\gamma}), \bar{\eta}(\dot{\gamma})$, and $\bar{\theta}(\dot{\gamma})$ can be calculated.

In case that $\lambda=0$, from (64) and (65) we obtain an equation for $f_{1}$ from which the following asymptotic solutions can be deduced:

$$
\begin{array}{ll}
f_{1} \rightarrow f_{10} & \text { for } \quad \dot{\gamma} \rightarrow 0, \lambda=0, \\
f_{1} \sim \dot{\gamma}^{-.417} & \text { for } \quad \dot{\gamma} \rightarrow \infty, \lambda=0 .
\end{array}
$$

So, by $(58,59,61,62)$ :

$$
\begin{array}{ll}
\eta \sim \dot{\gamma}^{-1} & \text { for } \quad \dot{\gamma} \rightarrow \infty, \lambda=0, \\
\theta \sim \dot{\gamma}^{-1.58} & \text { for } \quad \dot{\gamma} \rightarrow \infty, \lambda=0 .
\end{array}
$$




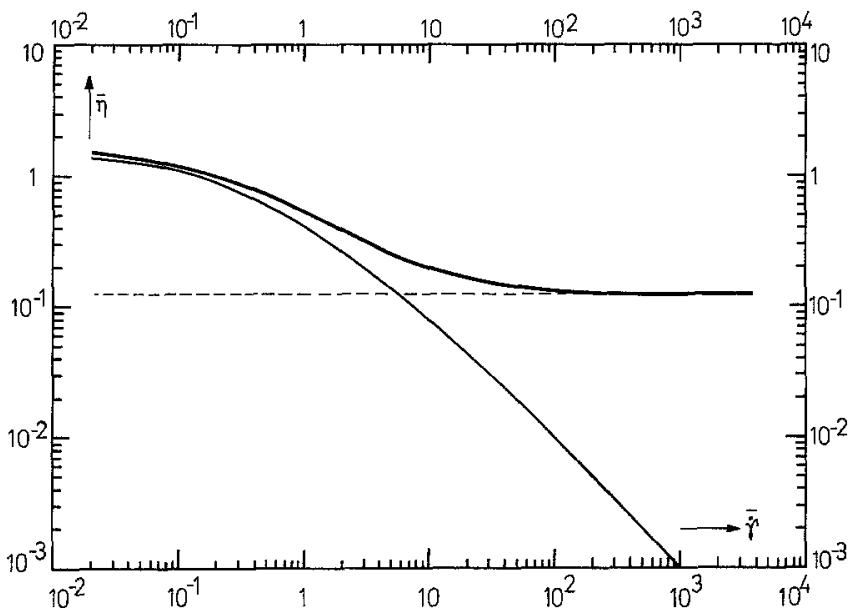

Fig. 2. Dimensionless viscosity as a function of the dimensionless shear rate with parameters $\lambda h_{10}=0.1$ and $f_{10}=0.8$ (— total; - fixed segments; - - - - free segments).

Thus, in the limiting case that our model reduces to the transient-network model, it predicts power-law behaviour for the fraction $f_{1}$ and the viscometric functions $\eta$ and $\theta$. The results (68) and (69) are in accordance with those given in [10]. In the case that $\lambda \neq 0, f_{1}$ will still approach zero for high values of $\dot{\gamma}$, whereas the viscometric functions will reach non-zero limiting values:

$$
\begin{aligned}
& \lim _{j \rightarrow \infty} \eta(\dot{\gamma})=\lambda N k T, \\
& \lim _{j \rightarrow \infty} \theta(\dot{\gamma})=2 \lambda^{2} N k T .
\end{aligned}
$$

Some results obtained by a numerical solution of eqs. $(61-65)$ are now presented. In figure 2 the dimensionless viscosity is given as a function of the dimensionless shear rate for certain parameter values. The contributions from fixed and free segments are given separately. At high shear rates the contribution from the free segments becomes larger than the network contribution, the decrease of which is due to the fact that the network is almost completely destroyed. Eq. (61) can be written in the following form:

$$
\begin{aligned}
& \bar{\eta}=\frac{h_{10}}{f_{10}}\left(\frac{f_{1}}{h_{1}}+\frac{\lambda f_{1}}{1-f_{1}}+\lambda\right) . \\
& \text { contri- contri- } \\
& \text { bution bution } \\
& \text { from from } \\
& \text { fixed free } \\
& \text { segment , segments }
\end{aligned}
$$

From eqs. (38-46) it can be shown that the contributions from fixed and free segments is as indicated. The free segments prevent the viscosity from going to zero at high shear rates.

In figure 3 the dimensionless first normal-stress coefficient is given as a function of the dimensionless shear rate. In figure 4 the structural variable $x=f_{1} / f_{10}$ is given versus the dimensionless shear rate. As expected, $x$ is seen to be a continuous decreasing function of $\bar{\gamma}$. The stress growth and the response of the variable $x$ upon the inception of a steady shear flow are obtained by numerically solving eqs. $(53-57)$ for the case that $\bar{\gamma}=0$ for $t<0$ and $\bar{\gamma}=$ constant for $t \geqq 0$, together with initial conditions (12) and (32). Some results, expressed by the dimensionless functions $\bar{\tau}(\bar{t}), \bar{\sigma}^{1}-\bar{\sigma}^{2}(\bar{t})$, and $x(\bar{t})$ are presented in figures 5,6 , and 7 for several

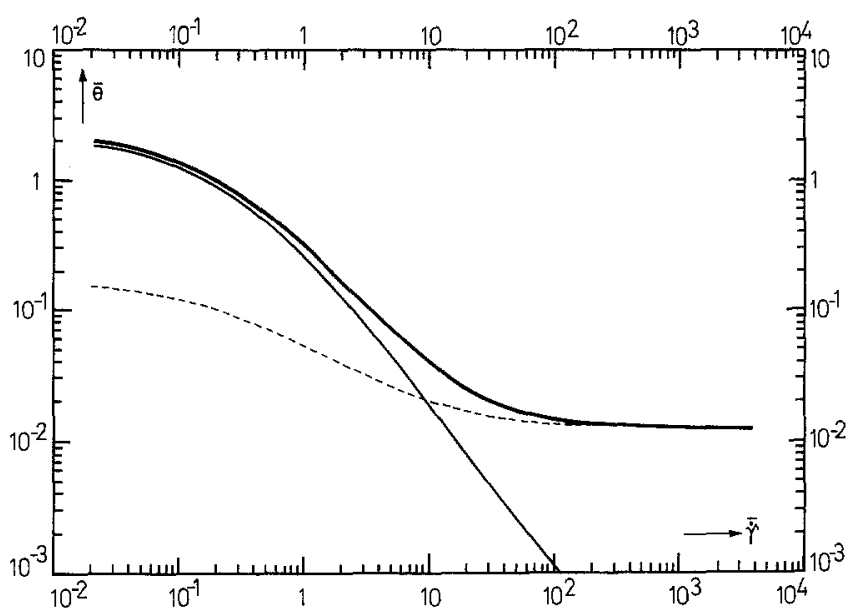

Fig. 3. Dimensionless first normal-stress coefficient as a function of the dimensionless shear rate with parameter $\lambda h_{10}=0.1$ and $f_{10}=0.8$ ( $($ total; - - fixed segments; --.- free segments).

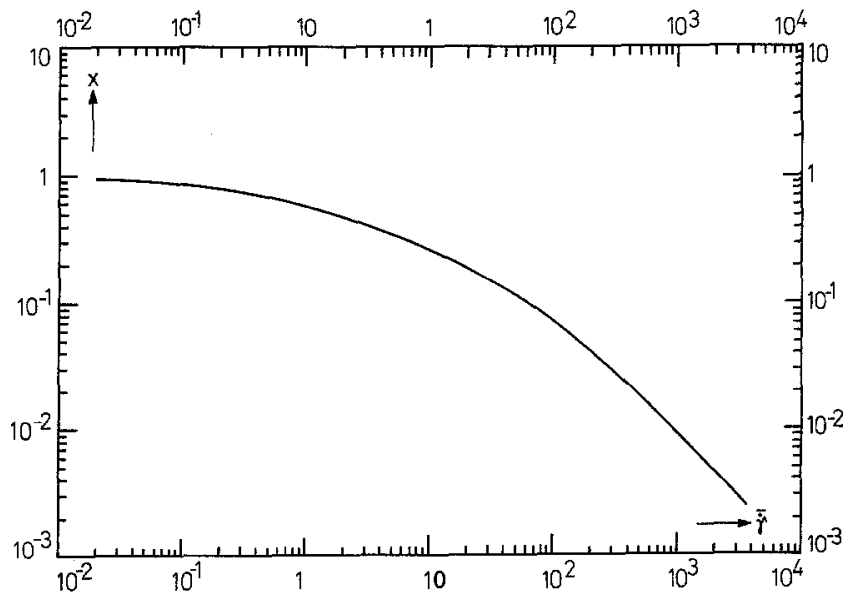

Fig. 4. The structural variable $x \equiv N_{1} / N_{10}$ as a function of the dimensionless shear rate with parameters $\lambda h_{10}=0.1$ and $f_{10}=0.8$. 


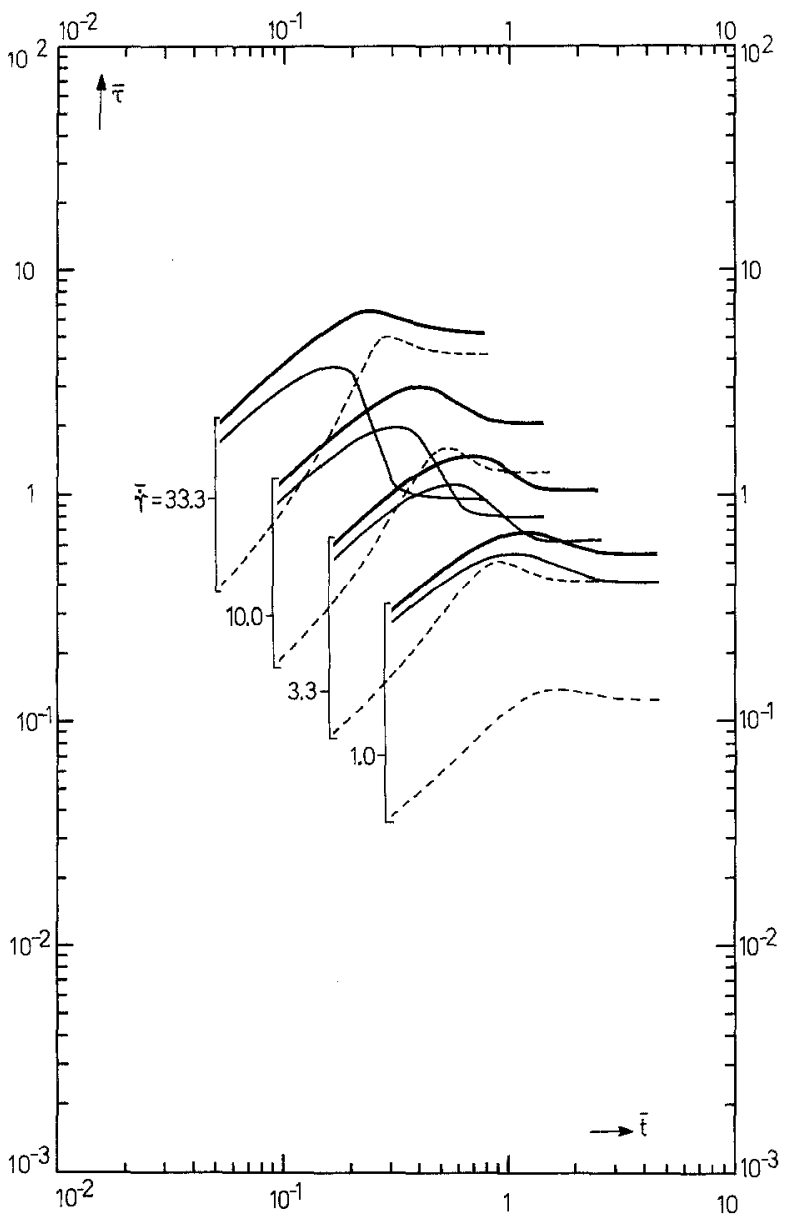

Fig. 5. Dimensionless shear stress as a function of the dimensionless time with parameters $\lambda h_{10}=0.1$ and $f_{10}=0.8$ at dimensionless shear rates $\bar{\gamma}=1.0,3.3,10.0,33.3$ (- total stress; _- fixed segments; - - -- free segments).

values of the dimensionless parameters $f_{10}, \lambda h_{10}$, and $\overline{\dot{\gamma}}$ Again, the contribution of the free segments becomes dominant at shear rates where $\bar{\gamma} \gtrsim 10$ and $\bar{t} \gtrsim 0.2$. Although both the fixed-segment and the free-segment contribution show overshoot, the ratio of maximum stress versus final stress is much higher for the network contribution. We see that introduction of the free chains causes flattening of the overall stress overshoot curves.

\section{Experimental}

\subsection{Introduction}

In the following sections model predictions for the stress growth in shear, the viscometric functions, and the linear viscoelastic behaviour are given for two

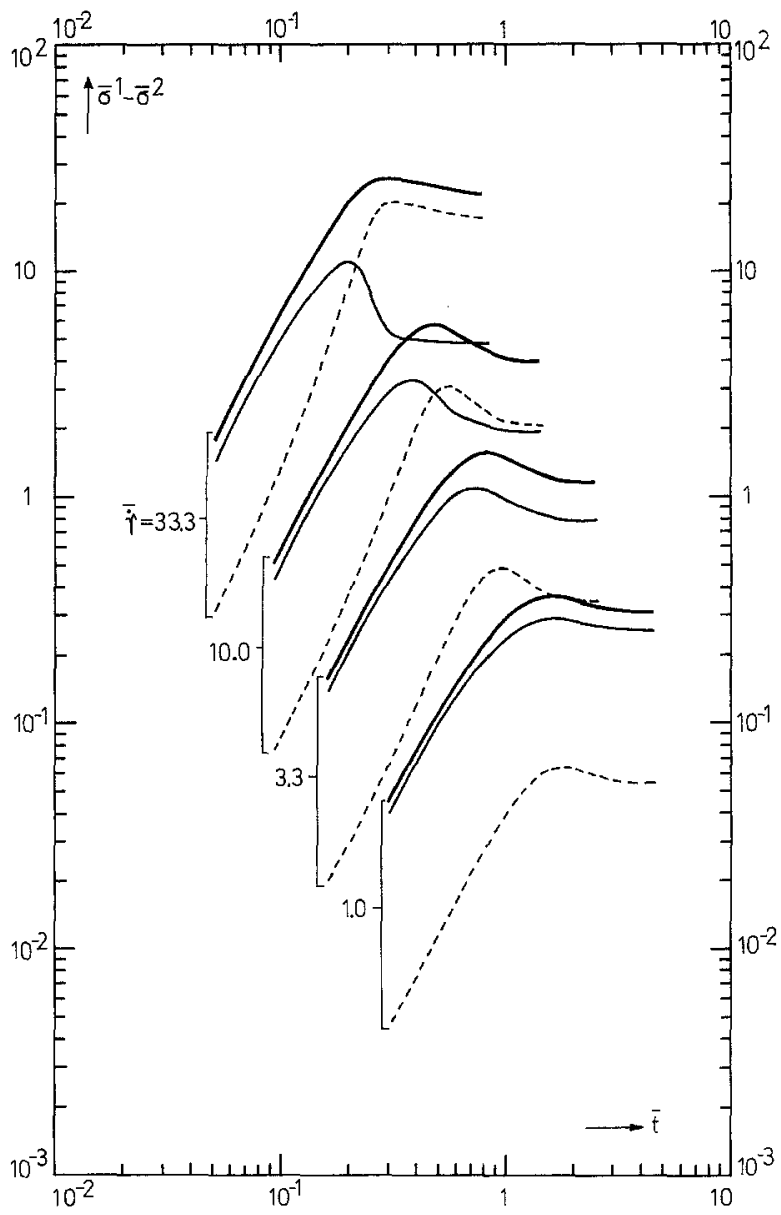

Fig. 6. Dimensionless normal stress as a function of dimensionless time with parameters $\lambda h_{10}=0.1$ and $f_{10}=0.8$ at dimensionless shear rates $\bar{\gamma}=1.0,3.3,10.0,33.3$ (— total stress; —_ fixed segments; - . - - f free segments).

polymer systems, namely an L.D. polyethylene melt and a solution of a S.I.S. 1107 block-copolymer in tetradecane. Since the model, as developed so far, does not possess a spectrum of relaxation times for either the fixed or the free segments, we do not expect a quantitative fit for these systems. Although further refinements are possible, our prime interest is to get some insight in the new concept of the model, viz. the simultaneous contribution of two different types of segments to the stress response.

\subsection{Model predictions compared with steady-state viscosity and transient-stress data on L.D. polyethylene}

We chose data reported in [11] and [12] for an L.D. polyethylene melt of type $\mathrm{A}$, obtained at $150^{\circ} \mathrm{C}$. The same data were used by Acierno et al. [9] for evaluation 


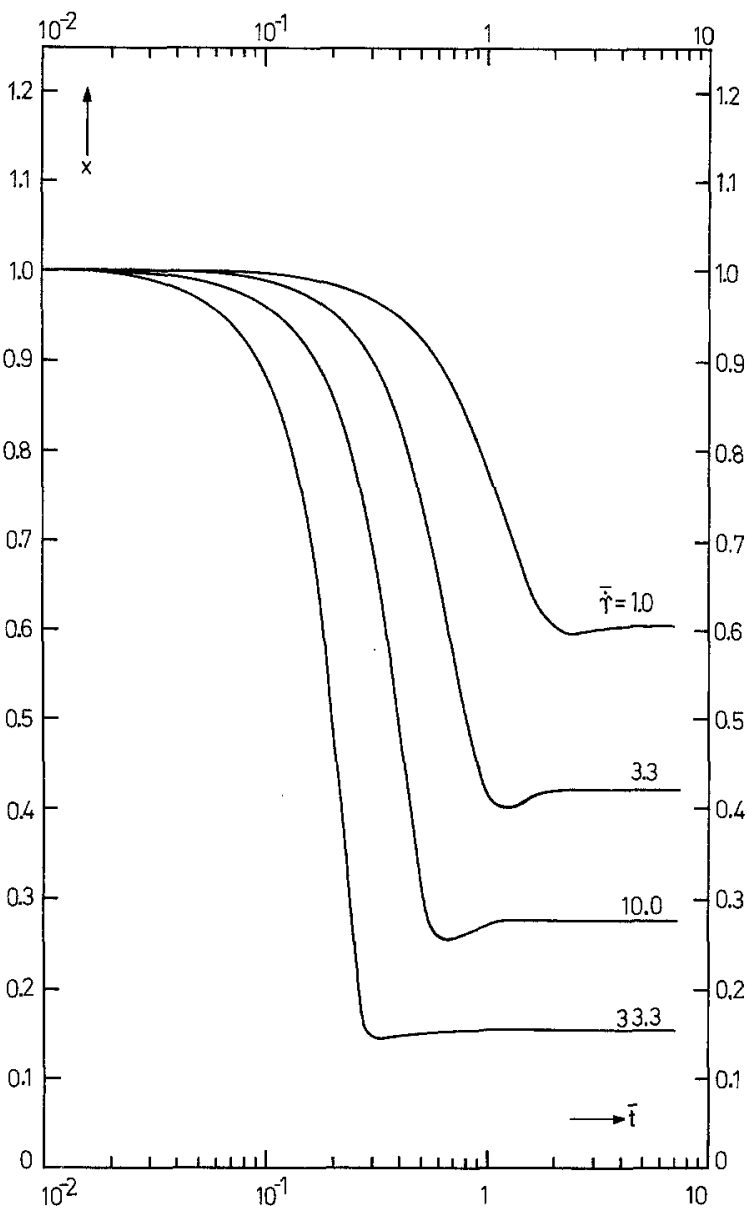

Fig. 7. The structural variable $x \equiv N_{1} / N_{10}$ as a function of the dimensionless time with parameters $\lambda h_{10}=0.1$ and $f_{10}=0.8$ at dimensionless shear rates $\bar{\gamma}=1.0,3.3,10.0,33.3$.

of a model based on a spectrum of structure-dependent relaxation times, in which a modified form of the kinetic functions (24) and (25) was used (see [10]).

The relaxation times and moduli that describe the linear viscoelastic behaviour as predicted by the model proposed here, see (29) and (30), are fit to correspond most favourably with the relaxation spectrum of the L.D. polyethylene melt as given in [11]. Subsequently, by inverting (29) and (30) the values of the model parameters, except for the non-linear parameter a (which was determined from the viscosity curve), are found (see table 1).

Using these values, from $(53-57,61,64,65)$ the model predictions for the shear viscosity as well as the stress growth in shear are obtained. These results are compared with experiments on the samples used. In figure 8 the shear viscosity is given as a function of the shear rate. In figure 9 shear-stress growth after incep-

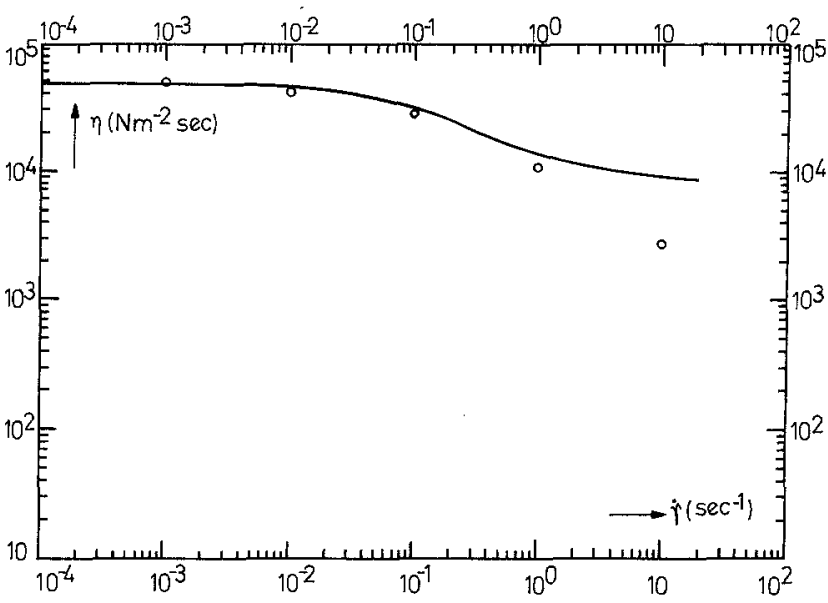

Fig. 8. The shear viscosity as a function of the shear rate for the L.D.P.E. melt, type $A$, at $423 \mathrm{~K}$ (O: measured; — calculated using the parameters in table 1 ).

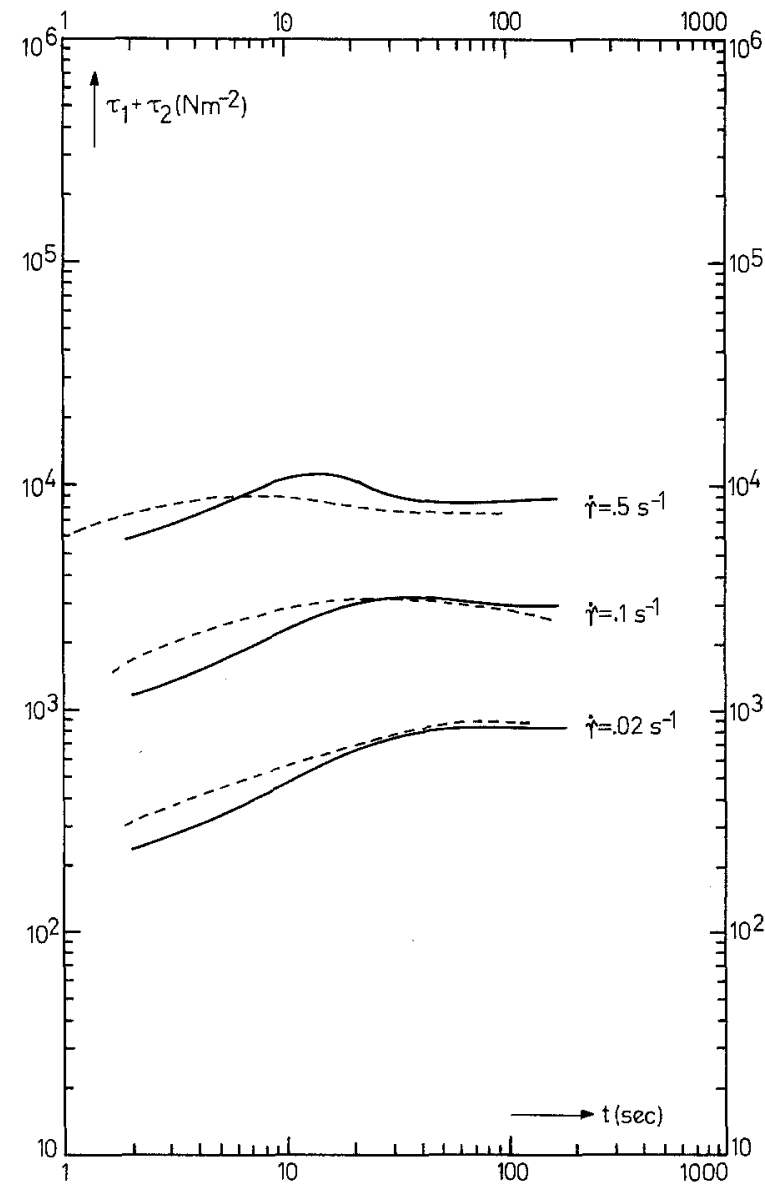

Fig. 9. The shear stress of an L.D.P.E.-melt, type A, at $423 \mathrm{~K}$, as a function of time, after inception of a steady-shear flow (-..- measured; - calculated using the parameters in table 1). 
Table 1. Model parameters following from dynamic measurements on L.D.P.E. type A systems at $150^{\circ} \mathrm{C}$

\begin{tabular}{ll}
\hline Relaxation times and moduli & Model parameters \\
\hline$\lambda_{1}=0.2 \mathrm{~s}$ & $\lambda=0.2 \mathrm{~s}$ \\
$\lambda_{2}=20 \mathrm{~s}$ & $h_{10}=0.05 \mathrm{~s}^{-1}$ \\
$G_{1}=4 \cdot 10^{4} \mathrm{~N} \mathrm{~m}^{-2}$ & $f_{10}=0.05$ \\
$G_{2}=2 \cdot 10^{3} \mathrm{~N} \mathrm{~m}^{-2}$ & $N=7 \cdot 10^{24} \mathrm{~m}^{-3}$ \\
& $a=0.2$ \\
\hline
\end{tabular}

tion of steady-shear flow is given as a function of time, for several values of the shear rate.

If one assumes the model to be valid, the model parameters given in table 1 reveal information about the structure of the systems at rest. Since $\lambda$ is a relaxation time connected with free draining molecular segments, a value of $0.2 \mathrm{~s}$ must be considered as high (see eq. (20)). The value of $h_{10}^{-1}$, i.c. $20 \mathrm{~s}$, may be considered as a characteristic time connected with the process of annihilation of network chains. It gives the mean life-time of network chains at rest.

Finally, $N$ and the ratio $f_{10}$ give the number of participating segments per unit volume and the fraction of chains that are part of the network, respectively. At rest, the system may be considered as a real network for about $5 \%$, whereas $95 \%$ consists of free molecules. In figure 10 the structural variable $x=f_{1} / f_{10}$ is given as a function of time upon inception of stady-shear flow.

In the theoretical curves the contribution of the free chains to the shear stress becomes significant at high shear rates. At these shear rates, however, the steadystate viscosity as predicted by the model tends to deviate from the experimental results. Two possible explanations are:

- the simplification made by considering two relaxation times instead of a spectrum forces one to attribute a part of the linear viscoelastic behaviour to the free segments, while in reality the effect of network motions might dominate;

- the free segments are modelled as Gaussian springs, while in the case of large stretching of segments, at high shear rates, the segments might be modelled by the inverse Langevin foce law, resulting in an effective relaxation time of free segments that is substantially lower (cf. eq. (20)).

\subsection{S.I.S. block-copolymers in tetradecane}

To test the model experimentally, we chose a solution of an S.I.S. block-copolymer in tetradecane. This system shows thermoreversible gelation: at low temperatures, gel formation takes place since the styrene

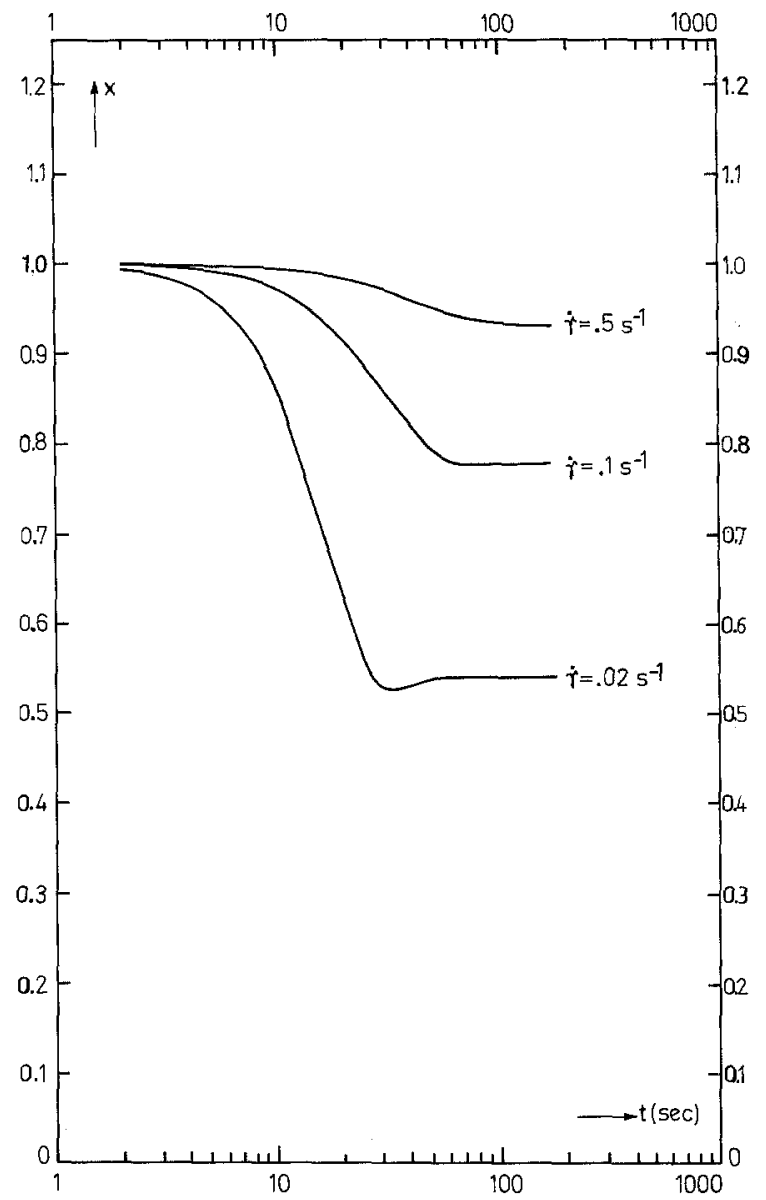

Fig. 10. The structural parameter $x \equiv N_{1} / N_{10}$ of an L.D.P.E. melt, type $\mathrm{A}$, at $423 \mathrm{~K}$, as a function of time, after inception of a steady-shear flow (Parameters used for calculation are given in table 1).

parts of the molecules, being insoluble in the solvent, will tend to associate; at higher temperatures, the gel dissolves and a relatively large number of molecules are expected not to be part of the network. So, one may expect this system to have a microstructure in accordance with the model as developed so far: a temporary network of segments of a well defined length, that may be either fixed of free. System properties are given in table 2.

The measurements were performed on a Rheometrics Mechanical Spectrometer, using cone-plate

Table 2. Properties of the S.I.S. solution in tetradecane

\begin{tabular}{lc}
\hline Mass concentration (wt.-\%) & 1.5 \\
Molecular weight & 160.000 \\
Molecular weight isoprene groups & 140.000 \\
Molecular weight styrene groups & 10.000 \\
$\bar{M}_{w} / \bar{M}_{n}$ & 1.25 \\
\hline
\end{tabular}




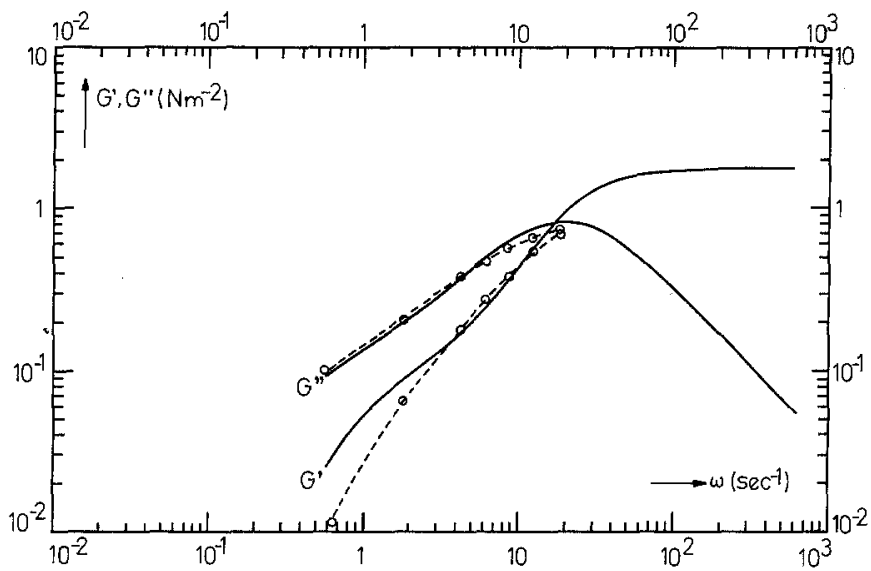

Fig. 11. Dynamic moduli of the 1.5 wt.-\% S.I.S. 1107 in $\mathrm{C}_{14} \mathrm{H}_{30}$ solution measured at $299.5 \mathrm{~K} \mathrm{(-.-} \mathrm{measured;} \mathrm{—-} \mathrm{set} \mathrm{of}$ times and moduli given in table 3 ).

Table 3. Model parameters following from measurements on an S.I.S.-block-copolymer solution (table 2)

\begin{tabular}{|c|c|c|}
\hline $\begin{array}{l}\text { Relaxation times } \\
\text { and moduli }\end{array}$ & $\begin{array}{l}\text { Model parameters } \\
\text { valid if } \dot{\gamma} \lesssim 10 \mathrm{~s}^{-1} \\
\text { set A }\end{array}$ & $\begin{array}{l}\text { Model parameters } \\
\text { valid if } \dot{\gamma} \gtrsim 20 \mathrm{~s}^{-1} \\
\text { set B }\end{array}$ \\
\hline $\begin{array}{l}\lambda_{1}=0.05 \mathrm{~s} \\
\lambda_{2}=0.9 \mathrm{~s} \\
G_{1}=1.6 \mathrm{~N} \mathrm{~m}^{-2} \\
G_{2}=0.1 \mathrm{~N} \mathrm{~m}^{-2}\end{array}$ & $\begin{array}{l}\lambda=0.05 \mathrm{~s} \\
h_{10}=1.1 \mathrm{~s}^{-1} \\
f_{10}=0.05 \\
N=4 \cdot 10^{20} \mathrm{~m}^{-3} \\
a=0.0016\end{array}$ & $\begin{array}{l}\lambda<10^{-4} \mathrm{~s} \\
h_{10}=0.04 \mathrm{~s}^{-1} \\
f_{10}=0.05 \\
N=4 \cdot 10^{20} \mathrm{~m}^{-3} \\
a=0.0016\end{array}$ \\
\hline
\end{tabular}

configuration with cone angle $0.04 \mathrm{rad}$, at a temperature of $299.5 \mathrm{~K}$.

Again, a set of two relaxation times and moduli is found from a global fit of the frequency-dependent elastic moduli (fig. 11).

From (29) and (30) the model parameters were determined; see table 3. A remarkable property of this system is that it shows shear-thickening behaviour in a certain range of shear rates. From the model predictions for the dimensionless shear viscosity (fig. 2) one may conclude that this behaviour cannot be predicted from the model by using the kinetic functions (24) and (25). A shear-rate dependent bond creation term should be introduced in order to obtain satisfying model predictions for any possible shear rate. The viscosity versus the shear rate, both experimentally determined and as predicted by the model, are given in figure 12. A second set of model parameters is introduced, from which a fit for higher shear rates, where $\dot{\gamma} \gtrsim 20 \mathrm{~s}^{-1}$, is found. However, it seems doubtful whether the information about the network structure that can be found from this set has physical significance as no fit for the whole range of shear rates is obtained.

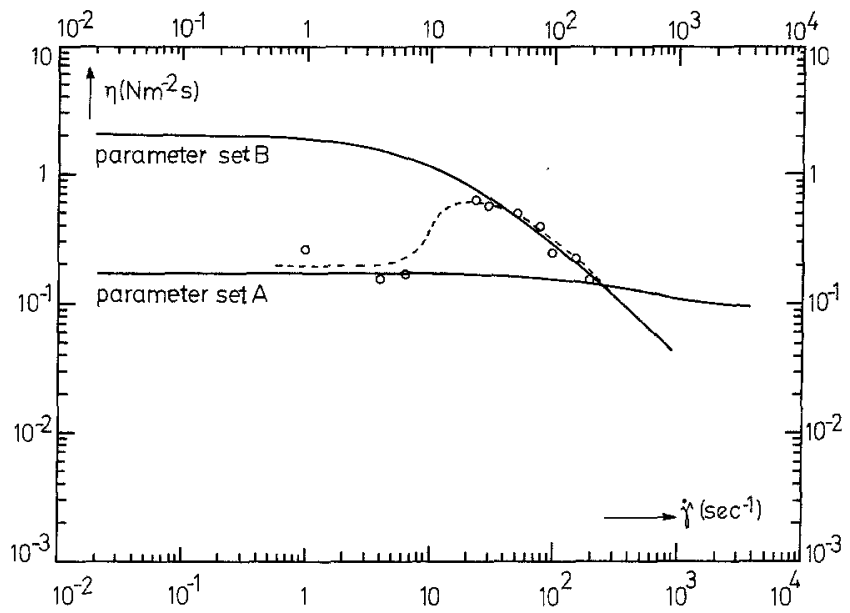

Fig. 12. The shear viscosity as a function of the shear rate for the 1.5 wt.- $\%$ S.I.S. 1107 in $\mathrm{C}_{14} \mathrm{H}_{30}$ solution, measured at $299.5 \mathrm{~K} \mathrm{(-.-} \mathrm{measured;} \mathrm{—} \mathrm{calculated} \mathrm{using} \mathrm{both} \mathrm{parameter}$ sets in table 3 ).

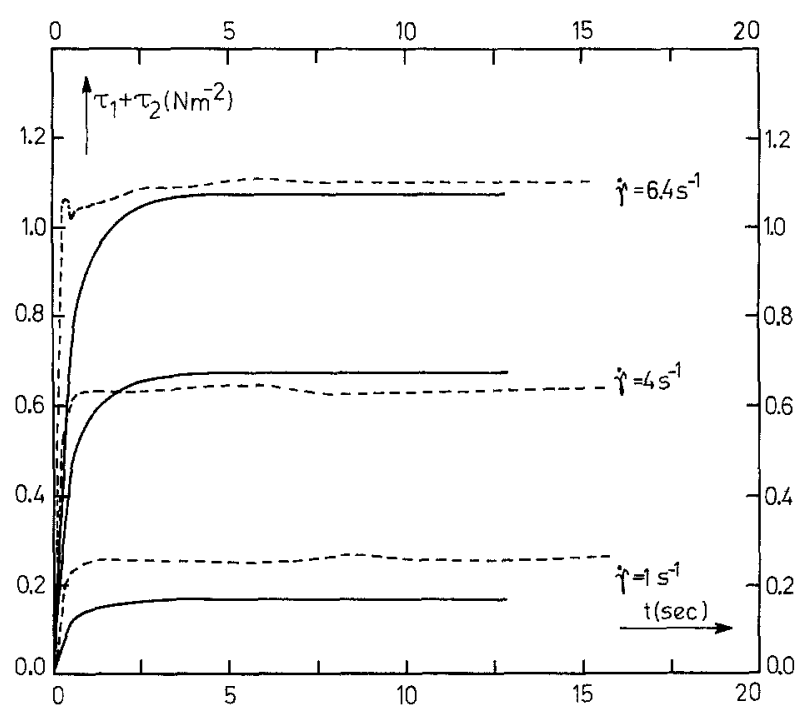

Fig. 13. The shear stress of a 1.5 wt.- $\%$ S.I.S. 1107 in $\mathrm{C}_{14} \mathrm{H}_{30}$ solution, after inception of a steady-shear flow (-..- measured; - calculated using parameter set $A$ in table 3 ).

In the range where $\dot{\gamma} \lesssim 10 \mathrm{~s}^{-1}$ model predictions have been achieved for the shear-stress response after inception of steady-shear flow. The model parameters found from linear dynamic measurements (table 3) were used; see figure 13.

If the present model is assumed to apply, table 3 yields information about the kinetic processes that take place as long as the shear rates do not exceed $10 \mathrm{~s}^{-1}$. The molecular relaxation time, $\lambda$, is found to be about $5 \%$ of the relaxation time $h_{10}^{-1}$, characterizing the kinetic process. 


\section{Conclusions}

The model presented in this paper shows how to combine the concepts characteristic for the temporarynetwork model and the bead-spring model by considering besides the fixed (network) segments also the free segments. The orientation distribution function of free segments follows from an equilibrium of forces acting on them. In this way the significance of two basic assumptions of the temporary-network model, mentioned in section 1, has been clarified. From the experimental results discussed in the preceding section if follows that it is not yet possible to obtain a unique interpretation of the kinetic processes that take place at a molecular level. In order to make further progress some modifications of the model are needed. The introduction of a spectrum of relaxation times as well as the use of other bond creation and annihilation functions would alter the model stress predictions and thereby its interpretation considerably. This is also the case when either the assumption of affinity of deformation or the linearity of the force law of the segment springs is modified.

\section{Acknowledgements}

Thanks are due to Prof. Dr. P. F. Mijnlieff for suggesting us the idea of considering the contribution of free segments within the concept of the transient-network model and for providing us with the S.I.S. blockcopolymer system, and to Mr. G. Johnston for carrying out a part of the experiments on the Rheometrics Mechanical Spectrometer.

\section{References}

1. Green, M. S., A. V. Tobolsky, J. Chem. Phys. 15, 651 (1947).

2. Lodge, A. S., Trans. Faraday Soc. 52, 120 (1956).

3. Yamamoto, M., J. Phys. Soc. Japan 11, 413 (1956).

4. Bird, R., O. Hassager, R. C. Armstrong, C. F. Curtiss, Dynamics of Polymeric Liquids, Vol. 2, John Wiley \& Sons (New York 1977).

5. Rouse, P. E. Jr., J. Chem. Phys. 21, 1272 (1953).

6. Zimm, B. H., J. Chem. Phys. 24, 269 (1956).

7. Lodge, A. S., Rheol. Acta 7, 379 (1968).

8. Jongschaap, R. J. J., J. Non-Newtonian Fluid Mech. 8, 183 (1981).

9. Marrucci, G., G. Titomanlio, G. C. Sarti, Rheol. Acta 12, 269 (1973).

10. Acierno, D., F. P. La Mantia, G. Marrucci, G. Titomanlio, J. Non-Newtonian Fluid Mech. 1, 147 (1976).

11. Meissner, J., J. Appl. Polym. Sci. 16, 2877 (1972)

12. Lodge, A. S., J. Meissner, Rheol. Acta 12, 41 (1973).

(Received December 10, 1982)

Authors' address:

Dr. R. J. J. Jongschaap et al. Department of Applied Physics

Twente University of Technology

P.O.B. 217

NL-7500 AE Enschede 